\title{
Momentum distribution of an electron scattered from a screened potential
}

\author{
D.H. Jakubassa-Amundsen \\ Physics Section, University of Munich, 8046 Garching, Germany
}

Received 21 May 1991; accepted for publication 27 June 1991

Communicated by B. Fricke

\begin{abstract}
The one-dimensional Fourier transform of an unbound eigenstate to a screened atomic potential is calculated and compared to the results for a Coulomb wave and a plane wave. It is found that for screened potentials with a strong central charge, the electron is in general not well localised in momentum space.
\end{abstract}

\section{Introduction}

The knowledge of exact electronic scattering states in the target field is of great importance for the calculation of electron loss in encounters with heavy target atoms, as well as for electron-induced ionisation at intermediate collision velocities. The use of plane waves or a perturbative treatment of the target field is not justified for heavy atoms, because the nuclear potentials are too strong, even when they are screened by an equal number of electrons [ 1 ]. For electron loss, the first Born approximation overestimates drastically the cross sections for e.g. Ne and Ar targets [2], and things get worse when the second Born term is included [3]. One must therefore either resort to coupled-channel calculations with large basis sets [2], or use perturbative approaches where the target field enters exclusively through the scattering amplitude or the scattering eigenfunction. The onedimensional Fourier transform of the eigenfunction occurs particularly if an additional peaking approximation is introduced which affects two of the three momentum components (the so-called transverse peaking approximation ). Such a peaking approximation implies that the momentum space wavefunction $\varphi_{k}(\boldsymbol{p})$ is strongly peaked around $\boldsymbol{p}=\boldsymbol{k}$ where $\boldsymbol{k}$ is the momentum of the scattered electron. Although true for plane waves and to a lesser extent for Coulomb waves, we show in the following that for screened potentials, this assumption should be treated with care. Atomic units $(\hbar=m=e=1)$ are used.

\section{Coulomb potential}

The scattering state of a pure Coulomb field $V(r)=-Z_{\mathrm{T}} / r$ where $Z_{\mathrm{T}}$ is the central charge, is the Coulomb wave which is known in closed form [4]. Since the Fourier transform of an incoming scattering state is identical to the complex conjugate Fourier transform of an outgoing scattering state, we can without loss of generality restrict ourselves to the latter, and define the one-dimensional Fourier transform by the following expression,

$G\left(p_{z}\right) \equiv \int \mathrm{d} \boldsymbol{p}_{\perp} \varphi_{\boldsymbol{k}}^{(-) *}(\boldsymbol{p})=\sqrt{2 \pi} \int_{-\infty}^{\infty} \mathrm{d} z \exp \left(\mathrm{i} p_{z} z\right) \psi_{\boldsymbol{k}}^{(-) *}\left(z \boldsymbol{e}_{z}\right)$, 
where $\psi_{\boldsymbol{k}}^{\prime-1}(\boldsymbol{r})$ is the outgoing wave in coordinate space. The choice of the $z$-direction $\boldsymbol{e}_{z}$ is arbitrary, and $\boldsymbol{p}$ denotes the components of the momentum $\boldsymbol{p}$ perpendicular to $\boldsymbol{e}_{z}$. Hence, $G\left(p_{z}\right)$ depends parametrically on $k$ and the angle $\vartheta$ between $\boldsymbol{k}$ and $\boldsymbol{e}_{z}$.

If a Coulomb wave is inserted for $\psi_{k}^{\mathbf{k}^{-1}}(\boldsymbol{r})$, an infinitesimal convergence factor has to be introduced into the r.h.s. of (1),

$$
\begin{gathered}
G^{c}\left(p_{z}\right)=\sqrt{2 \pi} N(k) \int_{-\infty}^{x} \mathrm{~d} z \exp \left[\mathrm{i}\left(p_{z}-k_{z}\right) z\right]_{1} F_{1}\left(\mathrm{i} \eta, 1, \mathrm{i}\left(k_{z} z+k|z|\right)\right) \exp (-\epsilon|z|), \\
N(k)=(2 \pi)^{-3 / 2} \exp (\pi \eta / 2) \Gamma(1-i \eta), \quad \eta=Z_{\mathrm{T}} / k, \quad \epsilon \rightarrow 0 .
\end{gathered}
$$

where $k_{z}=k \cos \vartheta,{ }_{1} F_{1}$ is the confluent hypergeometric function and $\Gamma$ the gamma function. The result is [5]

$$
G^{\mathrm{c}}\left(p_{z}\right)=\mathrm{i} \sqrt{2 \pi} N(k)\left[\frac{1}{p_{z}-k_{z}+\mathrm{i} \epsilon}\left(1+\frac{k_{z}+k}{p_{z}-k_{z}+\mathrm{i} \epsilon}\right)^{-\mathrm{i} \prime \prime}-\frac{1}{p_{z}-k_{z}-\mathrm{i} \epsilon}\left(1+\frac{k_{z}-k}{p_{z}-k_{z}-\mathrm{i} \epsilon}\right)^{-\mathrm{i} / \prime}\right] .
$$

We have verified from (3) that $(2 \pi)^{-3 / 2} \int_{-\infty}^{\infty} \mathrm{d} p_{z} G^{\mathrm{c}}\left(p_{z}\right)$ indeed equals the spatial wavefunction at the origin. $N(k)$.

The function $G^{\mathrm{c}}\left(p_{z}\right)$ has a branch point and a first-order pole in $p_{z}=k_{z}$, which in case of $k_{z}= \pm k$ turns into a $\delta$-function-type singularity. The latter property follows also immediately from (1) because for $k_{z}= \pm k_{,} F_{1}$ becomes unity in half the integration region. $G^{\mathrm{c}}\left(p_{z}\right)$ has in addition branch points in $p_{z}= \pm k$, but is smooth for $\left|p_{-}\right| \gg k$.

The plane-wave limit of $G\left(p_{z}\right)$, i.e. $\eta=0$, is readily obtained from (2) or (3).

$G^{\mathrm{pw}}\left(p_{z}\right)=\delta\left(p_{z}-k_{z}\right)$.

\section{Fully screened potential}

In the general case, an analytical solution for the scattering state $\psi_{k}^{(-)}(\boldsymbol{r})$ is no longer possible. For central potentials $V(r)$, it is common to make a partial wave expansion [4]

$\psi_{k}^{(-)}(\boldsymbol{r})=\frac{4 \pi}{(2 \pi)^{3 / 2}} \sum_{l m} \mathbf{i}^{\prime} \frac{1}{r} W_{l}(k, r) Y_{l m}(\hat{\boldsymbol{r}}) Y_{l m}^{*}(\hat{\boldsymbol{k}}) \exp \left(-\mathrm{i} \delta_{l}\right)$

where $Y_{l m}$ are spherical harmonics and $\delta_{l}$ is the phase shift. The function $W_{l}(k, r)$ obeys the radial Schrödinger equation

$\left(\frac{\mathrm{d}^{2}}{\mathrm{~d} r^{2}}+k^{2}-\frac{l(l+1)}{r^{2}}-2 V(r)\right) W_{l}(k, r)=0$.

When we restrict ourselves to energetic electrons (with $k^{2} / 2$ exceeding twice the mean target excitation energy), eq. (6) holds also for multielectron targets since exchange and orthogonality to the bound-state electrons can be neglected. $V(r)$ then consists of the atomic Hartree-Fock potential and the polarisation field [6].

With the help of (5) the Fourier transform of $\psi_{k}^{(-) *}$ is easily obtained,

$\varphi_{k^{\prime}}^{-) *}(\boldsymbol{p})=(2 \pi)^{-3 / 2} \int \mathrm{d} \boldsymbol{r} \exp (\mathrm{i} \boldsymbol{p} \cdot \boldsymbol{r}) \psi_{\boldsymbol{k}^{(-) *}}(\boldsymbol{r})=\frac{2}{\pi} \sum_{l m} \exp \left(\mathrm{i} \delta_{l}\right) Y_{l m}(\hat{\boldsymbol{k}}) Y_{l m}^{*}(\hat{\boldsymbol{p}}) \int_{0}^{\infty} r \mathrm{~d} r W_{l}(k, r) l_{l}(p r)$,

where $j_{i}$ is a spherical Bessel function.

For the evaluation of the one-dimensional Fourier transform $G\left(p_{z}\right)$ the momentum integral in $(1)$ is used. 
Writing $\mathrm{d} p_{\perp}$ in cylindrical coordinates $p_{\perp} \mathrm{d} p_{\perp} \mathrm{d} \varphi$ and noting that the only $\varphi$-dependence of (7) occurs in $Y_{l m}^{*}(\hat{\boldsymbol{p}})$, the integral over $\varphi$ is easily carried out and restricts the magnetic quantum number to $m=0$. The remaining integrals can be rearranged in the following way to give

$G\left(p_{z}\right)=\frac{1}{\pi} \sum_{T}(2 l+1) \exp \left(\mathrm{i} \delta_{l}\right) P_{l}(\cos \vartheta) \int_{0}^{\infty} r \mathrm{~d} r W_{l}(k, r) I_{l}\left(p_{z}, r\right)$,

$I_{l}\left(p_{z}, r\right)=\int_{0}^{\infty} p_{\perp} \mathrm{d} p_{\perp} P_{l}\left(p_{z} / p\right) j_{l}(p r)$,

where $P_{l}$ is a Legendre polynomial. With the help of the substitution $x=r p=r\left(p_{\perp}^{2}+p_{\beth}^{2}\right)^{1 / 2}$ and the symmetry relation $P_{l}(-x)=(-1)^{l} P_{l}(x)$, the integral $I_{l}$ can be evaluated analytically. One obtains $I_{0}=\cos \left(r p_{z}\right) / r^{2}$ and the simple recursion $r^{2} I_{t+1}=-\mathrm{d}\left(r^{2} I_{l}\right) / \mathrm{d}\left(r p_{z}\right)$, and hence

$$
\begin{aligned}
I_{l}\left(p_{z}, r\right)=(-1)^{\prime} \frac{1}{r^{2}} \frac{\mathrm{d}^{\prime}}{\mathrm{d}\left(r p_{z}\right)^{\prime}} \cos \left(r p_{z}\right) & =(-1)^{n} \frac{1}{r^{2}} \cos \left(r p_{z}\right), & & l=2 n, \\
& =(-1)^{n} \frac{1}{r^{2}} \sin \left(r p_{z}\right), & & l=2 n+1 .
\end{aligned}
$$

This formula is readily proven by inserting into (8) the recursion relations for $P_{l}$ and $j_{l}$ in terms of their derivatives [7], by performing integrations by part, and by using the differential equation for the Legendre polynomials.

The evaluation of $G\left(p_{z}\right)$ requires an analytical treatment [8,9] for large $r$ as well as for large $l$. For the first case, the $r$-integral in (8) is split into two parts, such that

$$
\int_{0}^{\infty} r \mathrm{~d} r W_{l}(k, r) I_{l}\left(p_{z}, r\right)=\int_{0}^{r_{\mathrm{m}}} r \mathrm{~d} r W_{l}(k, r) I_{l}\left(p_{z}, r\right)+\frac{1}{k} \int_{r_{\mathrm{m}}}^{\infty} r \mathrm{~d} r \sin \left(k r+\delta_{l}-\frac{1}{2} l \pi\right) I_{l}\left(p_{z}, r\right),
$$

where the first integral is calculated numerically (simultaneously with the calculation of $W_{l}$ from (6)) and $r_{\mathrm{m}}$ is chosen sufficiently large such that the radial wavefunction $W_{l}$ has attained its asymptotic form [4]. The second integral, valid for short-range potentials, can be evaluated analytically [5], and gives

$$
\begin{array}{ll}
-\frac{1}{2 k}\left\{\cos \delta_{l}\left[\operatorname{sgn}\left(q_{-}\right) \operatorname{si}\left(r_{\mathrm{m}}\left|q_{-}\right|\right)+\operatorname{si}\left(r_{\mathrm{m}} q_{+}\right)\right]+\sin \delta_{l}\left[\operatorname{ci}\left(r_{\mathrm{m}}\left|q_{-}\right|\right)+\operatorname{ci}\left(r_{\mathrm{m}} q_{+}\right)\right]\right\}, & l=2 n, \\
-\frac{1}{2 k} \operatorname{sgn}\left(p_{z}\right)\left\{\sin \delta_{l}\left[\operatorname{ci}\left(r_{\mathrm{m}}\left|q_{-}\right|\right)-\operatorname{ci}\left(r_{\mathrm{m}} q_{+}\right)\right]+\cos \delta_{l}\left[\operatorname{sgn}\left(q_{-}\right) \operatorname{si}\left(r_{\mathrm{m}}\left|q_{-}\right|\right)-\operatorname{si}\left(r_{\mathrm{m}} q_{+}\right)\right]\right\}, & l=2 n+1, \\
q_{ \pm}=k \pm\left|p_{z}\right|, &
\end{array}
$$

where $\operatorname{sgn}(q)$ denotes the sign of $q$ and si, ci are the sine and cosine integrals, respectively. Since ci $(x) \sim \ln x$ for $x \rightarrow 0, G\left(p_{z}\right)$ diverges logarithmically at $q_{-}=0$, i.e. $p_{z}= \pm k$. If, on the other hand, $V(r)$ contains a Coulombic contribution with the strength $k \eta$, a logarithmic term $\eta \ln (2 k r)$ has to be added to the argument of the sine in eq. (10). This term prevents the divergence at $p_{z}= \pm k$, such that there are only branch points left (see the discussion of (3)).

In order to handle the large- $l$ behaviour, we make use of the fact that for short-range potentials, $V(r)$ can be neglected for $l>l_{\max }$ in the Schrödinger equation (6) as compared to the centrifugal potential, when $l_{\max }$ is sufficiently large. This means that the high partial waves of $G\left(p_{z}\right)$ exhibit a plane-wave behaviour [9]. We therefore split the sum over $l$ in $(8)$ according to the following principle, 


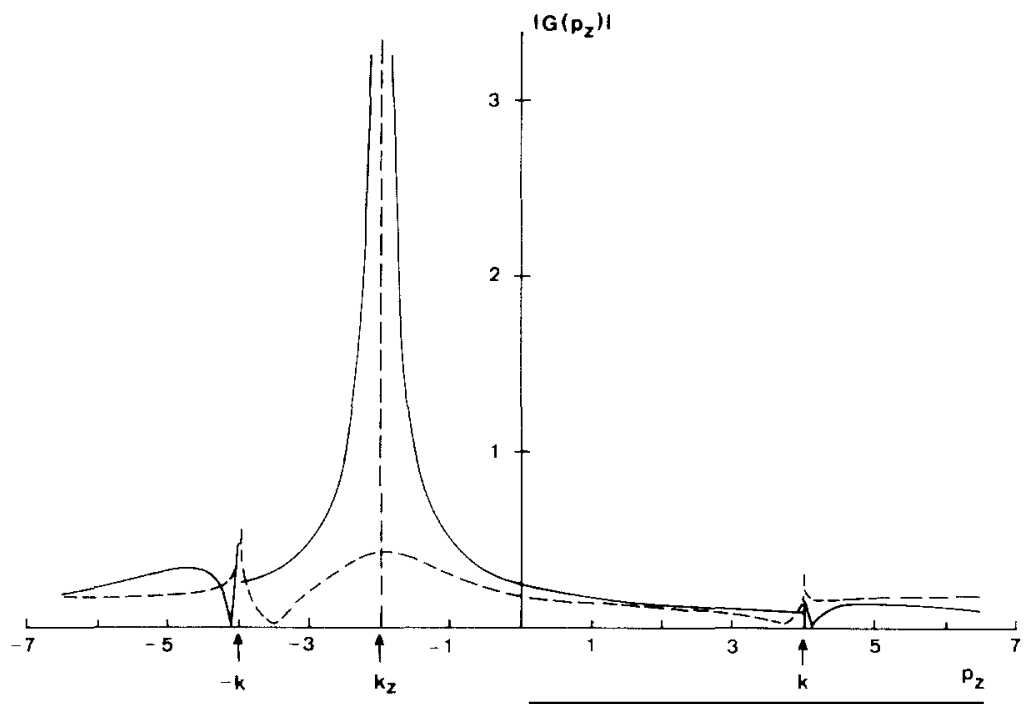

Fig. 1. Modulus of $G\left(k_{z}\right)$ in atomic units as a function of $k_{z}$ for an $\operatorname{Ar}$ target at $k=4$ and $\vartheta=120$. Plane wave: vertical dashed line at $p_{z}=-2$ : Coulomb wave: full line; and scattering state to screened potential: dashed line including the vertical line at $p_{z}=-2$. The positions of the branch points of the Coulomb wave at $p_{z}= \pm k$ and $k_{z}$ are marked by arrows.

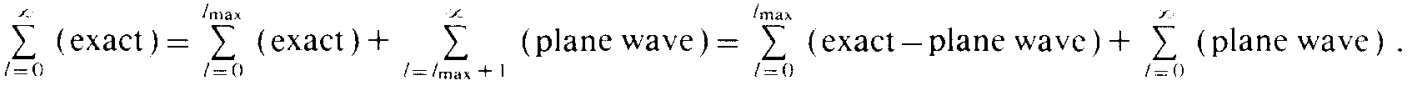

For a plane wave, the phase shifts are zero and $W_{l}^{p w}(k, r)=r j_{l}(k r)$, such that with the help of $(4), G\left(p_{z}\right)$ is written in the following form,

$G\left(p_{z}\right)=\frac{1}{\pi} \sum_{i=0}^{\prime \max }(2 l+1) P_{l}(\cos \vartheta)\left(\exp \left(\mathrm{i} \delta_{l}\right) \int_{0}^{x} r \mathrm{~d} r W_{l}(k, r) I_{l}\left(p_{z}, r\right)-\int_{0}^{x} r^{2} \mathrm{~d} r j_{l}(k r) I_{l}\left(p_{z}, r\right)\right)+\delta\left(p_{z}-k_{z}\right)$.

The plane-wave contribution for a given partial wave can again be evaluated analytically [5],

$$
\begin{aligned}
\int_{0}^{*} r^{2} \mathrm{~d} r j_{1}(k r) I_{l}\left(p_{z}, r\right) & \\
=(-1)^{n} \frac{\sqrt{\pi \theta\left(1-p^{2} / k^{2}\right)}}{k \Gamma(n+1)} & \times \frac{1}{2} \Gamma\left(n+\frac{1}{2}\right)_{2} F_{1}\left(n+\frac{1}{2},-n, \frac{1}{2}, p_{2}^{2} / k^{2}\right), \quad l=2 n, \\
& \times \frac{p_{z}}{k} \Gamma\left(n+\frac{3}{2}\right)_{2} F_{1}\left(n+\frac{3}{2},-n, \frac{3}{2}, p^{2} / k^{2}\right), \quad l=2 n+1,
\end{aligned}
$$

where the hypergeometric functions ${ }_{2} F_{1}$ reduce to polynomials, and $\theta$ is the Heaviside step function, making the r.h.s. of (14) vanish for $\left|p_{z}\right|>k$.

\section{Discussion}

For electrons scattering on an Ar target, $\left|G\left(p_{z}\right)\right|$ and $\left|G^{\mathrm{c}}\left(p_{z}\right)\right|$ are plotted in fig. 1 at an impact momentum of $k=4$ a.u., and the real part of these functions is given in fig. 2. For the Coulomb case we have used (3) 


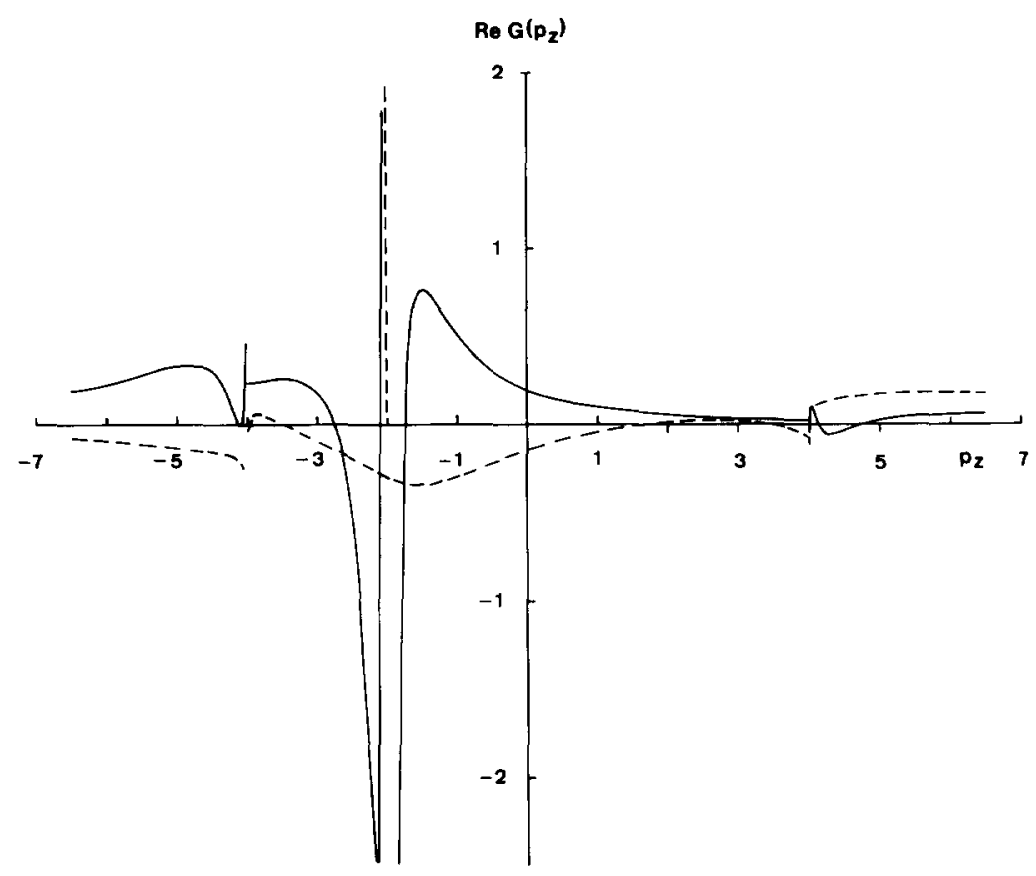

Fig. 2. Real part of $G\left(k_{z}\right)$ as a function of $k_{z}$ for an Artarget at $k=4$ and $\vartheta=120^{\circ}$. The curves have the same meaning as in fig. 1.

with $Z_{\mathrm{T}}=6$ which roughly corresponds to the effective charge seen by the valence electrons, such that $\eta=1.5$. For the screened case, the phase shifts and radial functions have been calculated as described previously [10]. Good convergence was obtained for $l_{\max }=20$ except close to the logarithmic singularities. In that region we took $l_{\max }=14$ where convergence was approximately reached (it deteriorated at higher $l$ due to numerical inaccuracies). The scattering angle $\vartheta$ was set to $120^{\circ}$, which is equivalent to $\vartheta=60^{\circ}$ due to the symmetry relation

$G\left(p_{z}, \vartheta\right)=G\left(-p_{z}, \pi-\vartheta\right)$,

which follows immediately from (3) and (13).

It is obvious from fig. 1 that for a plane wave as well as for a Coulomb wave, the momentum density is strongly concentrated near $p_{z}=k_{z}$, while for the scattering state of a screened field, it is more diffuse. For a target as heavy as $\mathrm{Ar}$, there are considerable contributions from the region $\left|p_{z}\right|>k$. This follows from inspection of the normalisation constant $N_{0}(k)$ of the $l=0$ radial wave, $\lim _{r \rightarrow 0}\left[W_{0}(k, r) / r\right] \exp \left(\mathrm{i} \delta_{0}\right)$ (which is identical to the integral of $G\left(p_{z}\right)$ over $\left.p_{z}\right)$, the magnitude of which is 5.4 for $k=4$ and hence much larger than one, the result from the $\delta$-contribution of $G\left(p_{z}\right) .\left|N_{0}(k)\right|$ decreases with $k$ towards unity (the plane-wave result), but very slowly (for $\mathrm{Ar},\left|N_{0}(10)\right|=3.4,\left|N_{0}(18)\right|=2.5$ ).

In contrast to the case of the screened-field scattering state which converges uniformly to a plane wave, the Coulomb wave shows a pathological behaviour when the central charge goes to zero [11], which does not allow for an expansion in terms of $Z_{\mathrm{T}}$. From fig. 2 and eq. (3) it follows that for any finite $\eta$ (i.e. $Z_{\mathrm{T}}$ ) and $\left|k_{z}\right| \neq k$ the Coulomb wave diverges at $p_{z}=k_{z}$ like $\left(p_{z}-k_{z}\right)^{-1-\mathrm{i} \eta}$ and not like a $\delta$-function. This is an additional indication that the limit $Z_{\mathrm{T}} \rightarrow 0$ differs from the result for $Z_{\mathrm{T}}=0$.

In conclusion we have shown that the one-dimensional Fourier transform $G\left(p_{z}\right)$ of a screened-potential scattering state has a very broad momentum distribution, with considerable contributions from $\left|p_{z}\right|$ exceeding the electronic impact velocity $k$. This feature is the more pronounced, the higher the nuclear target charge and the smaller $k$. The existence of the long-range tails of $G\left(p_{z}\right)$ is furthermore independent of the position angle $\vartheta$ of 
$\boldsymbol{k}$ with respect to the selected $z$-axis; however, the density near $p_{z}=k_{z}$ is enhanced for forward-backward directions as compared to $\vartheta \sim 90^{\circ}$.

The above results can readily be generalised to the three-dimensional Fourier transform $\varphi_{k}(p)$. In the fieldfree case of for weak fields, inspection of eq. (7) shows that the $r$-integral is peaked at $p=k$, and the partial wav $s$ add up coherently only if also the directions of $\boldsymbol{p}$ and $\boldsymbol{k}$ are the same. For strong fields with phase shifts cor siderably deviating from zero, and radial functions much different from Bessel functions, this coherence is vestroyed. Then the sharp peak at $\boldsymbol{p}=\boldsymbol{k}$ will be smeared out over a large region of momentum space.

\section{Acknowledgement}

This work was supported by the Gesellschaft für Schwerionenforschung, Darmstadt.

\section{References}

[1] H.R.J. Walters, J. Phys. B 8 (1975) L54.

[2] H. Ast, H.J. Lüdde and R.M. Dreizler, J. Phys. B 23 (1990) 2305.

[3] M. Kahle, Diploma Thesis, Munich (1991).

[4] L.D. Landau and E.M. Lifshitz, Quantum mechanics, Vol. III (Akademie-Verlag, Berlin, 1974) §§ 36, 134.

[5] I.S. Gradshteyn and I.M. Ryzhik, Table of integrals, series and products (Academic Press, New York, 1965) pp. 405, 747. 860.

[6] B.L. Jhanwar, S.P. Khare and K. Ashok Jr., J. Phys. B 11 (1978) 887.

[7] M. Abramowitz and I.A. Stegun, Handbook of mathematical functions (Dover, New York, 1965) pp. 232, 332,439

[8] G.E. Sneddon and I.B. Whittingham, J. Phys. B 23 (1990) 2227.

[9] D.H. Madison, R.V. Calhoun and W.N. Shelton, Phys. Rev. A 16 (1977) 552.

[10] D.H. Jakubassa, J. Phys. B 13 (1980) 2099.

[11] S. Okubo and D. Feldman. Phys. Rev. 117 (1960) 292. 